\title{
Alpha power is influenced by performance errors
}

\author{
JOSHUA CARP ${ }^{\mathrm{a}}$ AND REBECCA J. COMPTON ${ }^{\mathrm{b}}$ \\ ${ }^{a}$ Department of Psychology, University of Michigan, Ann Arbor, Michigan, USA \\ ${ }^{\mathrm{b}}$ Department of Psychology, Haverford College, Haverford, Pennsylvania, USA
}

\begin{abstract}
Error commission evokes changes in event-related potentials, autonomic nervous system activity, and behavior, presumably reflecting the operation of a cognitive control network. Here we test the hypothesis that errors lead to increased cortical arousal, measurable as changes in electroencephalogram (EEG) alpha band power. Participants performed a Stroop task while EEG was recorded. Following correct responses, alpha power increased and then decreased in a quadratic pattern, implying transient mental disengagement during the intertrial interval. This trend was absent following errors, which elicited significantly less alpha power than correct trials. Moreover, post-error alpha power was a better predictor of individual differences in post-error slowing than the error-related negativity (ERN), whereas the ERN was a better predictor of post-error accuracy than alpha power. These findings imply that changes in cortical arousal play a unique role in modulating post-error behavior.
\end{abstract}

Descriptors: EEG/ERP $<$ Measures Used, Cognition $<$ Content, Normal Volunteers $<$ Groups Studied

Error commission is associated with a suite of neural and behavioral changes, some or all of which may serve as monitoring signals used by the cognitive control system to maintain high levels of performance. Errors elicit event-related brain potentials such as the error-related negativity (ERN) and error positivity (Pe; Falkenstein, Hohnsbein, Hoormann, \& Blanke, 1991; Falkenstein, Hoormann, Christ, \& Hohnsbein, 2000; Gehring, Goss, Coles, Meyer, \& Donchin, 1993; Overbeek, Nieuwenhius, $\&$ Ridderinkhof, 2005). Errors are followed by increased autonomic arousal, as reflected by increased skin conductance and heart rate deceleration (Hajcak, MacDonald, \& Simons, 2003), and they potentiate the defensive startle reflex (Hajcak \& Foti, 2008). In addition, errors are often followed by behavioral changes such as slowed reaction time and changes in next-trial accuracy (e.g., Debener et al., 2005; Gehring et al., 1993; Ridderinkhof, Ullsperger, Crone, \& Nieuwenhuis, 2004). Here, we demonstrate that errors also lead to increased cortical arousal, as measured by changes in electroencephalogram (EEG) alpha power.

For more than 75 years, researchers have known that changes in the EEG power spectrum reflect changes in mental alertness (Davidson, Jackson, \& Larsen, 2000). Namely, relaxed or mentally drowsy states are associated with the presence of alpha waves, rhythmic oscillations around 8-14 Hz. Correspondingly, increases in mental activity or alertness lead to a relative decrease

We gratefully acknowledge the assistance of Alexandra Amen, Jessica Dodd, Stephanie Fineman, and Lorna Quandt in contributing to data collection.

Address reprint requests to: Rebecca J. Compton, Department of Psychology, Haverford College, 370 Lancaster Avenue, Haverford, PA 19041, USA. E-mail: rcompton@haverford.edu in power in the alpha frequency band. Thus, alpha power can serve as an inverse indicator of mental alertness or arousal.

More recently, researchers have measured phasic changes in alpha power to index transient changes in cortical activation associated with specific events (for reviews, see Klimesch, 1999; Klimesch, Sauseng, \& Hanslmayr, 2007; Pfurtscheller \& Lopes da Silva, 1999). Transient changes in alpha power can occur within the time frame of individual trials in performance tasks. For example, alpha power is reduced following a warning cue that signals an impending imperative stimulus, presumably because the cue increases arousal in anticipation of the target (Klimesch, Doppelmayr, Russegger, Pachinger, \& Schwaiger, 1998). Changes in alpha power can also be regionally specific. For example, cueing attention to either the left or right visual field leads to alpha power reductions over the contralateral posterior cortex (Sauseng et al., 2005; Thut, Nietzel, Brandt, \& Pascual-Leone, 2006).

Performance errors are thought to serve as alerting cues. According to several models of cognitive control, the detection of an error signals that performance adjustments are needed (e.g., Holroyd \& Coles, 2002; Ridderinkhof et al., 2004; Yeung, Botvinick, \& Cohen, 2004). In this sense, error detection may be similar to a warning cue that urges preparation for an upcoming trial. Therefore, reductions in alpha power should be observed following errors, just as has been shown following warning cues (Klimesch et al., 1998). Errors may also be emotionally arousing, as suggested by the fact that they heighten autonomic arousal and prime defensive reflexes (Hajcak \& Foti, 2008; Hajcak et al., 2003). These results also suggest that errors may provoke higher levels of cortical arousal as measured by alpha power in the EEG.

Despite the proliferation of studies examining ERP markers of error detection, to date there are no published reports examining 
changes in alpha power following errors. The present report tests the hypothesis that alpha power is decreased following errors compared to correct trials, indicating increased alertness after errors. In addition, we examine how post-error alpha power is related to error-related ERPs (the ERN and Pe) and behavioral changes. Comparing post-error changes in alpha power to error-related ERPs can help to determine whether both kinds of electrophysiological marker reflect the operation of a common control process or whether they tap unique aspects of post-error functioning. Likewise, examining the relationship between posterror alpha power and behavioral performance adjustments can help to determine the functional role of post-error changes in cortical arousal. Prior studies have only inconsistently linked the ERN and Pe with behavioral adjustments such as response slowing (e.g., Debener et al., 2005; Gehring et al., 1993; Gehring \& Fencsik, 2001; Hajcak et al., 2003), raising the possibility that other variables, such as post-error arousal, may yield additional predictive information about subsequent performance changes.

\section{Methods}

\section{Participants}

Participants were drawn from two separate studies that involved identical cognitive tasks. The first sample included 50 undergraduates and the second included 37. ERN and behavioral data from the first sample have already been reported (Compton et al., 2008), but that report did not examine post-error alpha data. For both samples, prescreening excluded people with neurological history, uncorrected visual defects, learning disability, or regular use of psychoactive medication. Six subjects were excluded from analysis due to noisy electrode leads at one or more sites, leaving a combined sample of $N=81$ (46 women, 35 men). Statistical analyses were carried out with sample as a factor, and no significant interactions involving sample emerged.

\section{Behavioral Task}

Participants completed a six-choice Stroop task on a Dell Dimension desktop computer running E-prime software. The task was to indicate the color of a target word using the first three fingers on each hand. The six color responses were red, orange, yellow, green, blue, and purple, mapped onto the fingers from left to right.

The task began with a practice block of 24 trials. Accuracy feedback was given after each practice trial, but not during the main trial blocks. The main experiment included either 12 blocks (sample 1) or 10 blocks (sample 2) of 66 trials per block. Each block included 30 incongruent trials (e.g., "red" in green font), 30 neutral trials (e.g., "dog" in green font), and 6 congruent trials (e.g., "green" in green font) in a randomly intermixed order.

Trial events included a 150 -ms presentation of the target word and then a blank screen that remained present until a key press or for a maximum of $2 \mathrm{~s}$. Following the key press, a blank screen was presented for $1100 \mathrm{~ms}$ until the onset of the next stimulus.

\section{EEG Recording and Signal Processing}

Electrodes were applied using an elastic cap (Quik-Caps) fitted with sintered $\mathrm{Ag} / \mathrm{AgCl}$ electrodes. Data were recorded continuously from eight scalp sites: Fz, FCz, Cz, Pz, F3, F4, C3, and C4. Signals were amplified by a NuAmps amplifier controlled by
Neuroscan software, with a sampling rate of $1000 \mathrm{~Hz}$ and a bandpass of $0.1-40 \mathrm{~Hz}(-3 \mathrm{~dB})$. Data were referenced online to the right mastoid and digitally re-referenced off-line to the average of left and right mastoids. Eye movements were monitored by electrodes placed above and below the left eye and at the outer canthus of each eye, and bipolar horizontal and vertical electrooculogram (EOG) channels were computed off-line.

Artifacts were addressed off-line in three steps. First, portions of the EEG record with large nonblink artifacts were manually excluded. Second, the effect of blinks was reduced using the Neuroscan software's regression-based algorithm. Finally, remaining artifacts in the EEG were identified using a $\pm 150 \mu \mathrm{V}$ threshold, and corresponding epochs were excluded. Following baseline correction, response-locked signal averaging was carried out separately for correct and error trials.

\section{Power Spectrum Quantification}

Power spectra were computed across the intertrial interval. EEG time series were divided into nonoverlapping 256-ms-long windows beginning at $0,256,512,768$, and $1024 \mathrm{~ms}$ postresponse. Power spectra were obtained for each window using the fast Fourier transform (FFT) using a cosine windowing method. This procedure yielded time-frequency representations of the intertrial interval with a resolution of $256 \mathrm{~ms}$ in the time domain and $4 \mathrm{~Hz}$ in the frequency domain. Spectra for each window were then averaged separately for correct and error trials. Statistical analyses were conducted using log-transformed mean power values in the $10-14 \mathrm{~Hz}$ frequency band.

\section{Results}

\section{Performance Data}

Accuracy and reaction time data were analyzed to characterize behavioral performance following errors versus correct responses. The mixed ANOVA on accuracy included previoustrial accuracy (error, correct) as a within-subjects factor and sample as a between-subjects factor. Accuracy was lower on trials following errors $(M=86 \%)$ than on trials following correct responses $(M=91 \%), F(1,79)=42.73, p<.0001$. A parallel analysis on correct-trial response times (RTs) found that responses were slower following errors $(M=741 \mathrm{~ms})$ than following correct responses $(M=654 \mathrm{~ms}), F(1,79)=83.98, p<.0001$. Overall, then, participants tended to become slower and less accurate following errors, compared to performance following correct trials.

We also examined whether Stroop interference was affected by accuracy on the previous trial, as might be the case if error detection caused the participant's attentional filter to be adjusted on the next trial (e.g., Ridderinkhof et al., 2004). Stroop interference was calculated as RT on incongruent trials minus RT on neutral trials, separately for trials that followed errors versus those that followed correct responses. Congruent trials were not included because there were too few post-error congruent trials for analysis. Overall, Stroop interference effects were highly significant both following errors $(M=74 \mathrm{~ms})$, one-sample $t(80)=5.67, \quad p<.0001$, and following correct responses $(M=55 \mathrm{~ms})$, one-sample $t(80)=11.31, p<.0001$. However, the difference between post-error and post-correct interference effects was not significant, paired-samples $t(80)=1.54, p>.12$.

Finally, we examined the relationships among individual differences in post-error accuracy, post-error slowing, and posterror Stroop interference. Error-related performance changes 
were quantified by an accuracy change score (post-error accuracy minus post-correct accuracy), an RT change score (post-error RT minus post-correct RT), and a Stroop interference change score (post-error Stroop interference minus post-correct Stroop interference). The RT and accuracy change scores were uncorrelated $(r=-.07)$, indicating that the tendency to slow down following errors was unrelated to changes in accuracy following errors. The Stroop interference change score was also unrelated to post-error accuracy change $(r=.13, p>.25)$, but it was positively correlated with the RTchange score $(r=.28, p<.02)$. The relationship between the RT change score and the Stroop interference change score remained consistent even when we statistically controlled for mean RT (partial $r=.28, p<.02$ ), indicating that the relationship was not confounded by individual differences in overall performance speed. In sum, participants who tended to slow down after errors also tended to exhibit more Stroop interference after errors, although they were no more or less likely to be accurate following errors.

\section{ERP Data}

We also analyzed error-related ERPs - the ERN and Pe-to replicate prior findings and to test correlations with behavioral measures of cognitive control. Figure 1 illustrates the responselocked ERP waveform at FCz, a representative site. For each participant, the ERN peak was defined as the most negative point within the interval -50 to $100 \mathrm{~ms}$ surrounding the response, and the Pe was defined as the most positive point within the 200-400-ms interval postresponse. Analyses of ERN and Pe data focus on the midline sites where these effects are typically observed.

ERN peak amplitudes were submitted to an ANOVA with trial accuracy (correct, error) and site ( $\mathrm{Fz}, \mathrm{FCz}, \mathrm{Cz}$, and $\mathrm{Pz})$ as within-subjects factors and sample as a between-subjects factor. Greenhouse-Geisser adjustments were applied to effects in this and all subsequent ANOVAs. As expected, the main effect of trial accuracy, $F(1,78)=125.20, p<.0001$, reflected greater negativity following errors $(M=-5.76 \mu \mathrm{V})$ than following correct responses $(M=-1.29 \mu \mathrm{V})$. The Accuracy $\times$ Site interaction, $F(3,234)=14.47, p<.001$, was due to greater accuracy differentiation at the FCz site compared to the other sites (see Table 1).

Pe amplitudes were also submitted to an ANOVA with trial accuracy, site, and sample as factors. The main effect of accuracy, $F(1,78)=156.05, p<.0001$, is due to higher amplitudes on error trials $(M=7.36 \mu \mathrm{V})$ compared to correct trials $(M=$ $-0.17 \mu \mathrm{V})$. The Accuracy $\times$ Site interaction, $F(3,234)=14.21$,

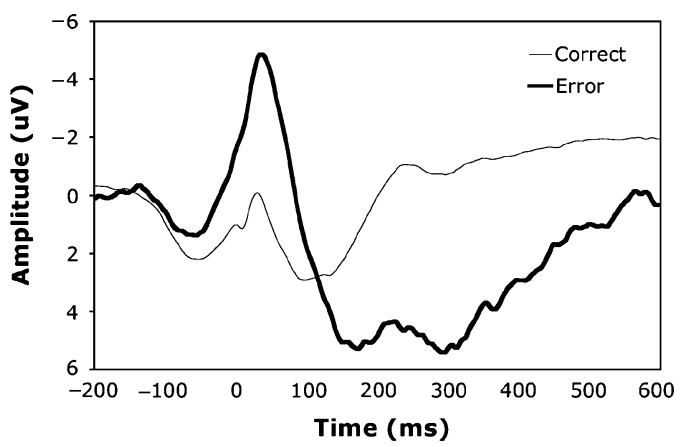

Figure 1. Grand-average waveform at FCz site, shown separately for correct and error trials. Time 0 is the time of the response.
Table 1. Amplitudes (in Microvolts) for ERN and Pe Peaks, Separately for Electrode Sites and Trial Types

\begin{tabular}{lccc}
\hline \hline & \multicolumn{3}{c}{ Trial type } \\
\cline { 2 - 4 } Electrode site & Correct & Error & Error - correct \\
\hline ERN & & & \\
$\mathrm{Fz}$ & -1.14 & -5.40 & -4.26 \\
$\mathrm{FCz}$ & -0.80 & -6.31 & -5.51 \\
$\mathrm{Cz}$ & -0.67 & -5.44 & -4.78 \\
$\mathrm{Pz}$ & -2.54 & -5.89 & -3.35 \\
$\mathrm{Pe}$ & & & 5.77 \\
$\mathrm{Fz}$ & 2.48 & 8.25 & 7.87 \\
$\mathrm{FCz}$ & 1.03 & 8.90 & 8.34 \\
$\mathrm{Cz}$ & -0.86 & 7.48 & 8.16 \\
$\mathrm{Pz}$ & -3.34 & 4.82 & \\
\hline \hline
\end{tabular}

$p<.001$, reflects better accuracy differentiation at the $\mathrm{FCz}, \mathrm{Cz}$, and $\mathrm{Pz}$ sites compared to the Fz site (see Table 1).

\section{Relationship between ERP Components and Behavioral Performance}

Between-subjects correlations were calculated to examine relationships among the error-related ERPs and behavioral performance measures. The ERN and Pe scores were represented as difference scores calculated at the FCz site. Higher ERN difference scores indicate higher peaks (more negative amplitudes) for error than correct trials, and likewise higher Pe difference scores indicate higher peaks (more positive amplitudes) for error than correct trials.

The error-related ERP variables were associated with posterror accuracy change, but not with post-error RT change. Higher ERN difference scores predicted higher post-error accuracy change scores $(r=.29, p<.01)$, as did the Pe difference scores $(r=.44, p<.001)$. The direction of the correlations indicates that bigger error-related ERP peaks were associated with relatively better performance following errors, compared to following correct trials. These two effects were independent of one another, as both the ERN and Pe difference scores remained significant predictors of post-error accuracy change in a multiple regression, multiple $r=.51, F(2,79)=13.74$; ERN: partial $r=.30, p<.01$; Pe: partial $r=.44, p<.001$. Both the ERN and the Pe difference scores also predicted higher overall accuracy (ERN: $r=0.35, p<.002$; Pe: $r=0.44, p<.001$ ). However, neither the ERN nor the Pe difference scores predicted individual differences in post-error RT slowing (ERN: $r=-.02$; Pe: $r=.02)$ or individual differences in post-error Stroop interference (ERN: $r=.05$; Pe: $r=-.08$ ). In sum, participants with more pronounced error-related ERP peaks were more likely to show improved accuracy following errors, but they were no more likely to slow down or show decreased Stroop interference following errors.

\section{Power Spectrum Data}

The ANOVA on log alpha power values included three repeated measures factors: trial accuracy (error, correct), time window (epochs beginning at 0, 256, 512, 768, and $1024 \mathrm{~ms}$ postresponse), and site (F3, Fz, F4, FCz, C3, Cz, C4, Pz). Sample was included as a between-subjects factor. For brevity, we report only effects involving the accuracy factor, as other effects are not relevant to the main hypothesis. 


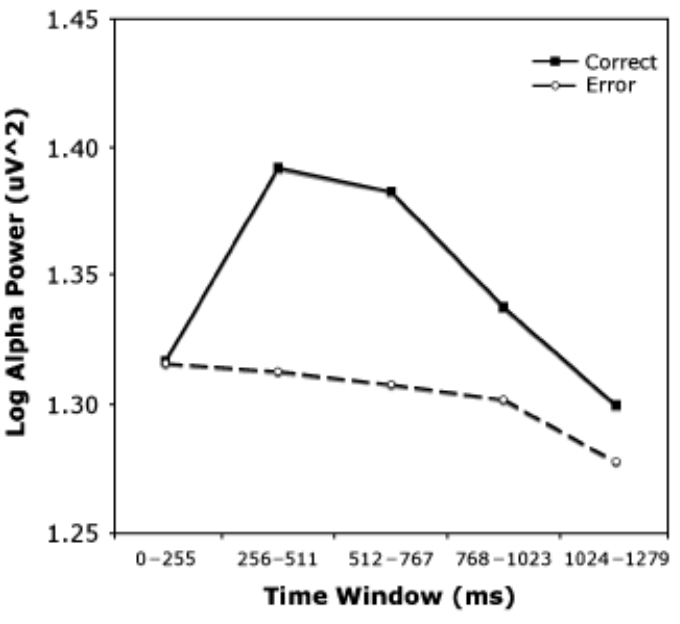

Figure 2. Alpha power following correct and error responses. Time 0 is the time of the response.

The main effect of accuracy, $F(1,79)=18.08, p<.0001$, revealed lower alpha power following errors $\left(M=1.30 \mu \mathrm{V}^{2}\right)$ than following correct trials $\left(M=1.35 \mu \mathrm{V}^{2}\right)$. The emergence of this effect over time is illustrated in Figure 2, Accuracy $\times$ Time Window, $F(4,316)=10.03, p<.0001$. A significant quadratic trend emerged following correct responses, $F(1,80)=52.08, p<.0001$, but the quadratic trend was absent following errors $(p>.30)$. In the 0 -ms time window, error and correct trials did not differ $(F<1)$, whereas in the three subsequent windows alpha power was significantly lower following errors than correct responses $(F \mathrm{~s}>8, p \mathrm{~s}<.005)$, and in the 1024-ms window the scores were marginally lower following errors than correct responses, $F(1,80)=3.69, p<.06$.

The effect of accuracy was evident across all sites, but it was most pronounced at the Pz site (see Table 2), Accuracy $\times$ Site, $F(7,553)=3.98, p<.005$. When the Pz site was removed from analysis, the Accuracy $\times$ Site interaction became nonsignificant ( $p>.35$ ), confirming that the interaction effect had been driven by the $\mathrm{Pz}$ site. Figure 3 illustrates the three-way interaction among accuracy, site, and time window, $F(28,2212)=4.58$, $p<.001$, with the interaction simplified by calculating an alpha reduction score (correct minus error) for each site and time window. Post-error alpha reduction was greater at the Pz site compared to other sites at early points in the intertrial interval, but in later windows the scores were comparable across sites.

Although the main effect of accuracy on alpha power was highly significant, these results were based on a sample that is fairly large in comparison to typical EEG/ERP studies. There-

Table 2. Postresponse Log Alpha Power (in Microvolts Squared) as a Function of Trial Type and Electrode Site

\begin{tabular}{lccc}
\hline \hline & \multicolumn{3}{c}{ Trial type } \\
\cline { 2 - 4 } Electrode site & Correct & Error & Correct - error \\
\hline F3 & 1.29 & 1.25 & 0.04 \\
Fz & 1.33 & 1.29 & 0.04 \\
F4 & 1.31 & 1.26 & 0.05 \\
FCz & 1.37 & 1.34 & 0.03 \\
C3 & 1.30 & 1.26 & 0.04 \\
Cz & 1.38 & 1.34 & 0.04 \\
C4 & 1.31 & 1.26 & 0.05 \\
Pz & 1.48 & 1.42 & 0.06 \\
\hline \hline
\end{tabular}

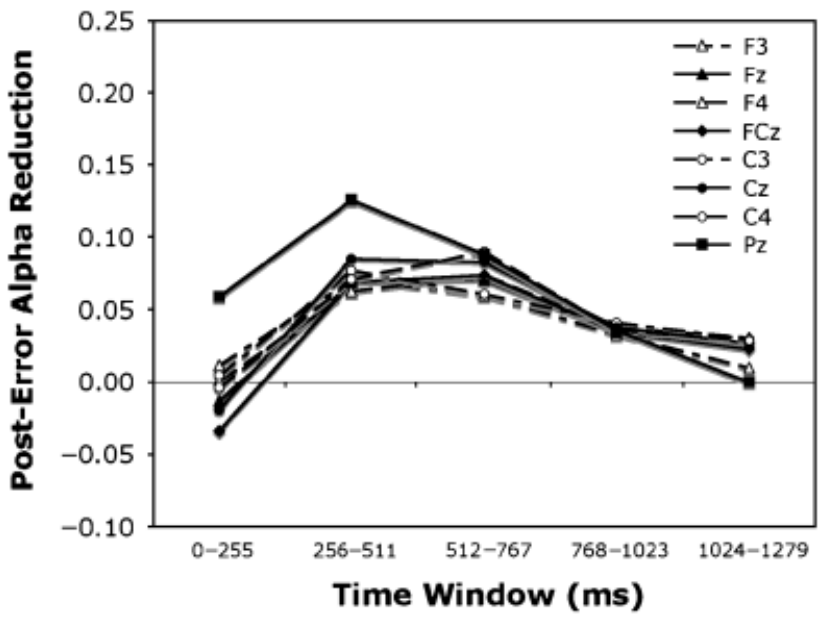

Figure 3. Alpha power reduction (correct minus error log alpha power) as a function of electrode site and time after the response.

fore, we calculated the sample size necessary to observe this main effect, based on the effect size of $\eta^{2}=.186$ from the present data set, assuming that the Type I error rate is controlled at .05 and assuming a correlation of .80 between repeated measures. The estimated sample size necessary to observe the main effect of accuracy at $95 \%$ power $(1-\beta)$ is 10 participants (Faul, Erdfelder, Lang, \& Buchner, 2007).

\section{Relationship between Alpha Power and Behavioral and ERP Variables}

To examine the relationship between individual differences in alpha power and individual differences in error-related behavior and ERPs, we included the behavioral and ERP measures as continuous predictors in the ANOVA on alpha data. These analyses of $\log$ alpha power included the repeated measures factors accuracy, time window, and site (as in the previous section), as well as the continuous predictor. The continuous predictor was either the accuracy change score, the RTchange score, the Stroop interference change score, the ERN difference score, or the Pe difference score (each entered in a separate analysis).

Results revealed that individual differences in the RT change score predicted the pattern of post-error alpha change, but none of the other ERP or behavioral variables did. Specifically, when the RTchange score was entered as the continuous predictor, the Accuracy $\times$ Time Window $\times$ RT Change interaction was significant, $F(4,316)=4.04, p<.007$. To better visualize this effect, we converted the continuous RT change scores into a categorical measure by dividing participants by a median split. Substituting this categorical predictor (RT change group) for the continuous predictor, the Accuracy $\times$ Time Window $\times$ RT Change group interaction remained significant, $F(4,316)=3.29, p<.02$. Means for the interaction are depicted in Figure 4.

To best characterize the source of the three-way interaction, we decomposed the interaction in several ways. First, we examined data from correct and error trials separately. For correct trials, the Time Window $\times$ RT Change interaction was not significant $(F<1)$, but for error trials the interaction was significant, Time Window $\times$ RT Change, $F(4,316)=4.89, p<.002$. Thus, the three-way interaction was driven by the error trials. We then examined the trends separately for participants in the low and high RTchange groups. Among the participants in the lower half of the RT change distribution - that is, participants who were 

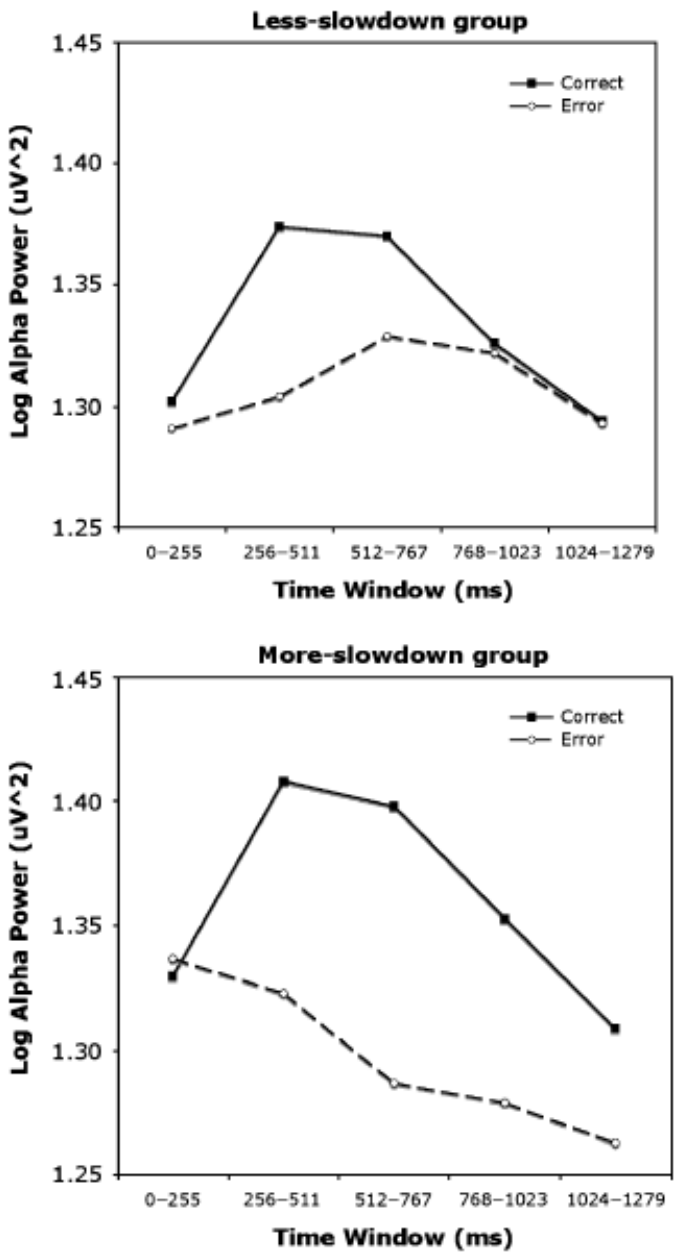

Figure 4. Alpha power following correct and error responses, separately for participants who differed in reaction-time (RT) slowing following errors. The top panel illustrates the pattern of alpha power in participants who tended to slow down less after errors (scoring at or below the median RT change score) and the bottom panel illustrates the pattern in participants who tended to slow down more following errors (scoring above the median RT change score). Time 0 is the time of the response.

less likely to slow down following errors - alpha power after errors followed a marginally significant quadratic pattern, $F(1,40)=3.74, p<.07$, with no linear component $(F<1)$. In contrast, among participants in the upper half of the RT change distribution - that is, participants who were more likely to slow down following errors - alpha power after errors decreased over time following a linear trend, $F(1,39)=8.59, p<.007$, with no significant quadratic component $(F<1)$. Finally, to determine the time window at which individual differences in post-error slowing predicted the pattern of alpha data, we examined the data from each time window separately. The accuracy $\times$ RT change interaction was significant for the 512-, 768-, and 1024-ms windows $(F \mathrm{~s}>4, p \mathrm{~s}<.05)$ but not for the 0 - or $256-\mathrm{ms}$ windows $(F \mathrm{~s}<1)$. In other words, alpha effects related to post-error performance speed were most evident later in the intertrial interval.

Analyses yielded no additional significant effects when the accuracy change score, Stroop interference change score, ERN difference score, or Pe difference score was entered as the continuous predictor. Therefore, individual differences in the errorrelated ERPs and individual differences in post-error accuracy appeared to be unrelated to the pattern of alpha power change.

\section{Full-Spectrum Analysis}

Because predictions concerned error-related effects in the alpha range, results in other frequency bands will not be presented in depth in this report. However, we present a brief exploratory analysis of the entire power spectrum. We used complex demodulation (Müller et al., 1994) to compute time-frequency (T-F) surfaces for the intertrial interval. T-F surfaces were obtained separately for errors and correct trials for each participant and were log-transformed. Next, we compared each entry of the error and correct T-F surfaces using the $t$ statistic, yielding one $t$ surface for the entire sample. Finally, the critical $t$ value for the $t$ surface was adjusted using the false discovery rate correction for multiple comparisons (Genovese, Lazar, \& Nichols, 2002). Figure 5 shows the error-related $t$ surface based on data from all sites and thresholded at an FDR-corrected $\alpha$ value of .05. In agreement with the windowed Fourier analysis reported above, this analysis revealed a strong modulation of alpha power by response accuracy: postresponse alpha power was reduced following errors relative to correct trials. The T-F analysis also showed relative increases in theta-, high beta-, and gamma-band power after errors.

\section{Discussion}

The main finding of this study is that errors are followed by significantly less alpha power compared to correct trials, indicating higher cortical arousal after a mistake than after a correct response. After correct responses, alpha power followed a pronounced quadratic trend, first increasing and then decreasing as participants presumably experienced transient mental disengagement during the intertrial interval. In contrast, this quadratic pattern of alpha power was absent on error trials. These results imply that mental alertness was maintained during the intertrial interval following errors, in contrast to the disengagement following correct trials.

Alpha power measures predicted different aspects of posterror behavioral performance than did ERP measures. Individual differences in post-error alpha power were related to post-error changes in speed but not to post-error changes in accuracy. Participants with greater post-error slowing showed significant alpha reduction after errors; conversely, among participants with less post-error slowing, post-error alpha patterns were more similar to post-correct alpha patterns, particularly toward the end of the intertrial interval. In contrast, individual differences in the ERN and Pe predicted post-error accuracy, as participants with higher ERN/Pe amplitudes were more likely to show improved accuracy following errors (relative to correct trials); however, the ERN and Pe did not predict post-error speed. This latter finding appears to contradict some prior studies reporting an association between the ERN amplitude and nexttrial slowing. However, the studies that have most reliably found positive associations between these two variables have focused on trial-to-trial variation within an individual (e.g., Debener et al., 2005; Gehring et al., 1993). Factors that account for withinsubject (trial-to-trial) variation are conceptually distinct from factors that account for between-subjects variation (individual differences). Our results indicate that between-subjects variation in post-error alpha power (but not the ERN or Pe amplitude) can predict between-subjects variation in post-error slowing.

Together, the results from individual differences analyses imply that there are at least two separable constellations of neural 


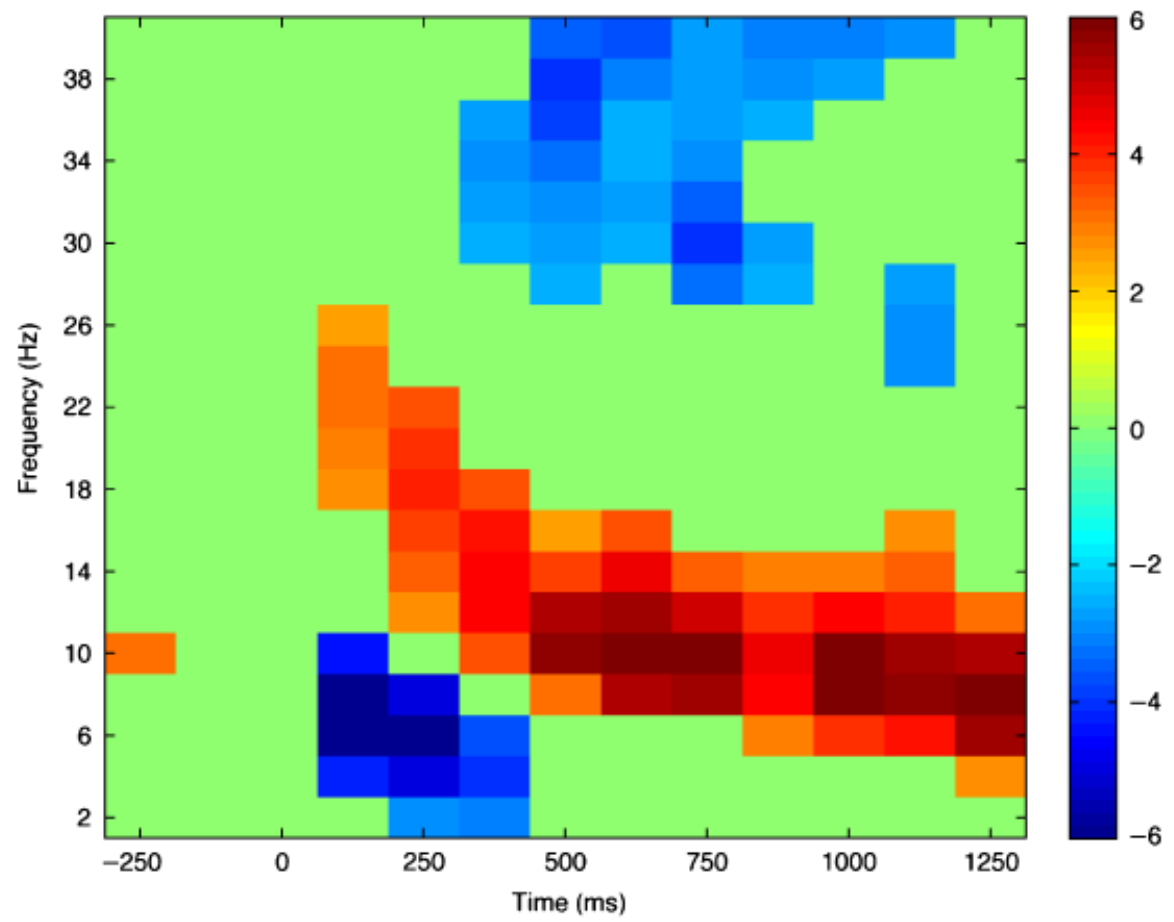

Figure 5. Error-related time-frequency $t$ surface based on data from all sites. The color scale represents $t$ units. Positive $t$ values (warm colors) indicate greater power for correct trials than for errors; negative $t$ values (cool colors) indicate greater power for errors than for correct trials. Time 0 is the time of the response.

and behavioral changes following errors. Post-error changes in performance accuracy tap an adaptive control system that yields performance improvements following mistakes. The ERN and Pe reflect error-monitoring processes that are closely tied to this adaptive control system, as argued previously by other researchers (e.g., Holroyd \& Coles, 2002). In contrast, post-error changes in speed are not necessarily adaptive, at least when considered from the perspective of individual differences. Although researchers have often assumed that post-error slowing represents an adaptive compensatory process (e.g., Ridderinkhof et al., 2004), we found that individuals who slowed down more following errors were neither more nor less likely to improve accuracy following errors. That is, post-error changes in speed and post-error changes in accuracy appear to be independent of one another. Participants who slowed down more after errors also tended to show more Stroop interference after errors, further undermining the assumption that post-error slowing is adaptive.

Rather than reflecting the engagement of an adaptive control system, post-error slowing may reflect the engagement of an arousal system. Alpha power has long been associated with arousal, with greater alpha power indicating lower states of arousal (Davidson et al., 2000). In the present data set, post-error alpha power was lower among those who slowed down more following errors; in other words, higher post-error arousal predicted greater behavioral slowing after errors. Although the reasons for this relationship are unclear at present, it may be that increased post-error arousal is associated with momentary confusion or uncertainty, which in turn leads to slower responses on the next trial. Alternatively, increased post-error arousal may reflect ongoing cognitive processing that interferes with the steps needed to prepare effectively for the next trial.

This study does not directly address the neural pathway that connects error commission with changes in alpha power, but prior theorizing offers a framework for interpretation. In a theoretical review, Aston-Jones and Cohen (2005) argued that the brainstem locus coeruleus (LC), the source of norepinephrine inputs to the forebrain and a known modulator of cortical arousal, can play an important role in modifying ongoing task performance. Descending projections from the anterior cingulate cortex make contact with neurons in the LC. Because the anterior cingulate cortex is recruited following errors in choice tasks (e.g., Dosenbach et al., 2006; Kerns et al., 2004; Mathalon, Whitfield, \& Ford, 2003), error commission could activate the LC via the cingulate. This LC activation could in turn lead to widespread arousal throughout the forebrain areas that receive norepinephrinergic projections from the LC. Such increased norepinephrinergic arousal could lead to either better or worse task performance, depending on tonic baseline levels of arousal (Aston-Jones \& Cohen, 2005). Stimulation of the LC is known to affect the EEG power spectrum (e.g., Berridge \& Foote, 1991), so it is plausible that error commission could lead to changes in alpha power through this cingulate-LC-norepinephrine route.

Future studies should continue to investigate the post-error alpha power phenomenon using other performance tasks. The present study used a six-choice Stroop task, which may be more challenging than other superficially similar tasks used in the cognitive control literature. For example, many other studies have used the Eriksen flankers task (Eriksen \& Eriksen, 1974), which typically includes only two response choices (e.g., press right button for rightward arrow, left button for leftward arrow) and four possible stimulus arrays (e.g., rightward and leftward arrow targets flanked by congruent or incongruent arrow arrays). Our task included six possible response choices and 66 possible stimulus arrays. An error on this more complex task may lead to different kinds of post-error cognitive changes than an error on simpler tasks like the flankers task. After making an error, 
participants in the present study may have needed to mentally review the stimulus-response mapping, which was less intuitive than on the typical flankers task. In addition, because the next trial could be any one of dozens of possible stimulus displays, different next-trial preparation processes may be involved than in the flankers task, in which only four next-trial options need to be anticipated (cf. Egner, 2007; Mayr, Awh, \& Laurey, 2003; Nieuwenhuis et al., 2006). Such task-specific factors could account for why errors on our task were followed by less accurate performance and no improvements in resolving interference, counter to what might have been predicted based on current models of cognitive control. Ultimately, any viable model of post-error cognitive control will need to be flexible enough to encompass a wide range of performance tasks.

Another potential avenue for future research is to better pinpoint the neural sources of arousal-related changes following errors. In the present study, the overall reduction in alpha power after errors was evident across all recorded sites, but the effect was most pronounced at the parietal site, particularly within early time windows following the error. Because other studies often find a posterior maximum for alpha measurements (Nunez, Wingeier, \& Silberstein, 2001; see also Feige et al., 2005; Moosmann et al., 2003), it is not surprising that errors modulated alpha power most dramatically at the parietal site. Regional differences were not the main focus of this study, and we sampled from a relatively small number of sites. In addition, scalp EEG is a notoriously poor localizer of component brain systems. Future studies using other imaging methods may better address the extent to which changes in arousal following errors are restricted to certain neural locations or distributed across the brain in a relatively undifferentiated manner.

Future studies may also examine the functional significance of EEG oscillations in other frequency bands following errors.
Although our main focus in this report was on error-related changes in alpha power, the full-spectrum analysis demonstrated post-error changes elsewhere on the frequency spectrum as well. This analysis showed that whereas power was decreased following errors in the alpha range, power was increased in the theta and gamma ranges. The results in the theta band are consistent with prior reports of changes in theta activity coinciding with the ERN (e.g., Luu, Tucker, Derryberry, Reed, \& Poulsen, 2003; Luu, Tucker, \& Makeig, 2004; see Yeung, Bogacz, Holroyd, Nieuwenhuis, \& Cohen, 2007, for alternative interpretations). Post-error increases in the gamma frequency range have not, to our knowledge, been previously reported, although a recent study found changes in gamma power following negative feedback (Papo et al., 2007). The gamma-band data from the present study should be interpreted with caution because our low-pass filter cutoff was set at $40 \mathrm{~Hz}$, and common definitions of gamma describe a band from 30 up to $100 \mathrm{~Hz}$. Nevertheless, given recent research on the potential role of gamma oscillations in attention (Jensen, Kaiser, \& Lachaux, 2007), error-related changes in the gamma band may merit future investigation.

In sum, the results from this study demonstrate a novel effect of error commission on cortical arousal, as measured by power in the alpha frequency band of the EEG. The power of alpha oscillations recorded from frontal, central, and parietal electrodes was reduced following errors, relative to correct responses. Further, error-related changes in alpha power predicted posterror behavioral slowing across subjects, an effect that was not paralleled in analyses of error-evoked ERPs. These findings highlight the broader relevance of EEG spectral analysis to the study of cognitive control. Future research should continue to expand the taxonomy of behavioral and physiological responses to errors and to delineate the relationships between these responses and the cognitive control system.

\section{REFERENCES}

Aston-Jones, G., \& Cohen, J. D. (2005). An integrative theory of locus coeruleus-norepinephrine function: Adaptive gain and optimal performance. Annual Review of Neuroscience, 28, 403-450.

Berridge, C. W., \& Foote, S. L. (1991). Effects of locus coeruleus activation on electroencephalographic activity in neocortex and hippocampus. Journal of Neuroscience, 11, 3135-3145.

Compton, R. J., Robinson, M. D., Ode, S., Quandt, L. C., Fineman, S. L., \& Carp, J. (2008). Error-monitoring ability predicts daily stress regulation. Psychological Science, 19, 702-708.

Davidson, R. J., Jackson, D. C., \& Larson, C. L. (2000). Human electroencephalography. In J. T. Cacioppo, L. G. Tassinary, \& G. G. Berntson (Eds.), Handbook of Psychophysiology (2nd ed., pp. 27-52). Cambridge, UK: Cambridge University Press.

Debener, S., Ullsperger, M., Siegel, M., Fiehler, K., von Cramon, D. Y., \& Engel, A. K. (2005). Trial-by-trial coupling of concurrent electroencephalogram and functional magnetic resonance imaging identifies the dynamics of performance monitoring. Journal of Neuroscience, 25, 11730-11737.

Dosenbach, N. U. F., Visscher, K. M., Palmer, E. D., Miezin, F. M., Wenger, K. K., Kang, H. C., et al. (2006). A core system for the implementation of task sets. Neuron, 50, 799-812.

Egner, T. (2007). Congruency sequence effects and cognitive control. Cognitive, Affective, and Behavioral Neuroscience, 7, 380-390.

Eriksen, B. A., \& Eriksen, C. W. (1974). Effects of noise letters upon the identification of a target letter in a nonsearch task. Perception \& Psychophysics, 16, 143-149.

Falkenstein, M., Hohnsbein, J., Hoormann, J., \& Blanke, L. (1991). Effects of crossmodal divided attention on late ERP components. II. Error processing in choice reaction tasks. Electroencephalography and Clinical Neurophysiology, 78, 447-455.
Falkenstein, M., Hoormann, J., Christ, S., \& Hohnsbein, J. (2000). ERP components on reaction errors and their functional significance: A tutorial. Biological Psychology, 51, 87-107.

Faul, F., Erdfelder, E., Lang, A.-G., \& Buchner, A. (2007). G*Power 3: A flexible statistical power analysis program for the social, behavioral, and biomedical sciences. Behavior Research Methods, 39, 175-191.

Feige, B., Scheffler, K., Esposito, F., Di Salle, F., Hennig, J., \& Seifritz, E. (2005). Cortical and subcortical correlates of electroencephalographic alpha rhythm modulation. Journal of Neurophysiology, 93, 2864-2872.

Gehring, W. J., \& Fencsik, D. E. (2001). Functions of the medial frontal cortex in the processing of conflict and errors. Journal of Neuroscience, 21, 9430-9437.

Gehring, W. J., Goss, B., Coles, M. G. H., Meyer, D. E., \& Donchin, E. (1993). A neural system for error detection and compensation. Psychological Science, 4, 385-390.

Genovese, C. R., Lazar, N. A., \& Nichols, T. E. (2002). Thresholding of statistical maps in functional neuroimaging using the false discovery rate. NeuroImage, $15,870-878$.

Hajcak, G., \& Foti, D. (2008). Errors are aversive: Defensive motivation and the error-related negativity. Psychological Science, 19, 103-108.

Hajcak, G., McDonald, N., \& Simons, R. F. (2003). To err is autonomic: Error-related brain potentials, ANS activity, and post-error compensatory behavior. Psychophysiology, 40, 895-903.

Holroyd, C. B., \& Coles, M. G. H. (2002). The neural basis of human error processing: Reinforcement learning, dopamine, and the errorrelated negativity. Psychological Review, 109, 679-709.

Jensen, O., Kaiser, J., \& Lachaux, J.-P. (2007). Human gamma-frequency oscillations associated with attention and memory. Trends in Neurosciences, 30, 317-324. 
Kerns, J. G., Cohen, J. D., MacDonald, A. W., Cho, R. Y., Stenger, V. A., \& Carter, C. S. (2004). Anterior cingulate conflict monitoring and adjustments in control. Science, 303, 1023-1026.

Klimesch, W. (1999). EEG alpha and theta oscillations reflect cognitive and memory performance: A review and analysis. Brain Research Reviews, 29, 169-195.

Klimesch, W., Doppelmayr, M., Russegger, H., Pachinger, T., \& Schwaiger, J. (1998). Induced alpha band power changes in the human EEG and attention. Neuroscience Letters, 244, 73-76.

Klimesch, W., Sauseng, P., \& Hanslmayr, S. (2007). EEG alpha oscillations: The inhibition-timing hypothesis. Brain Research Reviews, 53, 63-88.

Luu, P., Tucker, D. M., Derryberry, D., Reed, M., \& Poulsen, C. (2003). Electrophysiological responses to errors and feedback in the process of action regulation. Psychological Science, 14, 47-53.

Luu, P., Tucker, D. M., \& Makeig, S. (2004). Frontal midline theta and the error-related negativity: Neurophysiological mechanisms of action regulation. Clinical Neurophysiology, 115, 1821-1835.

Mathalon, D. H., Whitfield, S. L., \& Ford, J. M. (2003). Anatomy of an error: ERP and fMRI. Biological Psychology, 64, 119-141.

Mayr, U., Awh, E., \& Laurey, P. (2003). Conflict adaptation effects in the absence of executive control. Nature Neuroscience, 6, 450-452.

Moosmann, M., Ritter, P., Krastel, I., Brink, A., Thees, S., Blankenburg, F., et al. (2003). Correlates of alpha rhythm in functional magnetic resonance imaging and near infrared spectroscopy. NeuroImage, $20,145-158$.

Müller, M. M., Rockstroh, B., Berg, P., Wagner, M., Elbert, T., \& Makeig, S. (1994). SSR-modulation during slow cortical potentials. In C. Pantev (Ed.), Oscillatory event-related brain dynamics (pp. 325342). New York: Plenum Press.

Nieuwenhuis, S., Stins, J. F., Posthuma, D., Polderman, T. J. C., Boomsma, D. I., \& De Geus, E. J. (2006). Accounting for sequential trial effects in the flanker task: Conflict adaptation of associative priming? Memory \& Cognition, 34, 1260-1272.
Nunez, P., Wingeier, B. M., \& Silberstein, R. B. (2001). Spatial-temporal structures of human alpha rhythms: Theory, microcurrent sources, multiscale measurements, and global binding of local networks. $\mathrm{Hu}$ man Brain Mapping, 13, 125-164.

Overbeek, T. J. M., Nieuwenhuis, S., \& Ridderinkhof, K. R. (2005). Dissociable components of error processing: On the functional significance of the Pe vis-a-vis the ERN/Ne. Journal of Psychophysiology, 19, 319-329.

Papo, D., Douiri, A., Bouchet, F., Bourzeix, J.-C., Caverni, J.-P., \& Baudonniére, P.-M. (2007). Feedback modulates gamma oscillations in a hypothesis testing paradigm. Brain Research, 1141, 147-153.

Pfurtscheller, G., \& Lopes da Silva, F. H. (1999). Event-related EEG/ MEG synchronization and desynchronization: Basic principles. Clinical Neurophysiology, 110, 1842-1857.

Ridderinkhof, K. R., Ullsperger, M., Crone, E. A., \& Nieuwenhuis, S. (2004). The role of medial frontal cortex in cognitive control. Science, 306, 443- 447.

Sauseng, P., Klimesch, W., Stadler, W., Schabus, M., Doppelmayr, M., Hanslmayr, S., et al. (2005). A shift of visual spatial attention is selectively associated with human EEG alpha activity. European Journal of Neuroscience, 22, 2917-2926.

Thut, G., Nietzel, A., Brandt, S. A., \& Pascual-Leone, A. (2006). $\alpha$-band electroencephalographic activity over occipital cortex indexes visuospatial attention bias and predicts visual target detection. Journal of Neuroscience, 26, 9494-9502.

Yeung, N., Bogacz, R., Holroyd, C. B., Nieuwenhuis, S., \& Cohen, J. D. (2007). Theta phase resetting and the error-related negativity. Psychophysiology, 44, 39-49.

Yeung, N., Botvinick, M. M., \& Cohen, J. D. (2004). The neural basis of error detection: Conflict monitoring and the error-related negativity. Psychological Review, 111, 931-959.

(Received May 8, 2008; ACCePted June 18, 2008) 